ZOOLOGIA 27 (3): 413-424, June, 2010

doi: $10.1590 /$ S1984-46702010000300014

\title{
Neotropical Pentatomidae (Insecta: Hemiptera: Heteroptera) of the collection of Massimiliano Spinola preserved in the "Museo Regionale de Scienze Naturali", Turin, Italy
}

\author{
Jocélia Grazia \& Luiz A. Campos
}

Departamento de Zoologia, Universidade Federal do Rio Grande do Sul. Avenida Bento Gonçalves 9500, 91501-970 Porto Alegre, Rio Grande do Sul, Brazil.E-mail: jocelia@ufrgs.br; luiz.campos@ufrgs.br

\begin{abstract}
One hundred and forty specimens of Neotropical pentatomids of the Spinola collection were examined and identified and are listed in the present work. Lectotypes are designated for Arvelius gladiator Spinola, 1837; Arvelius laciniatus Spinola, 1837; Audinetella bipunctata Spinola, 1850; Ochlerus cinctus Spinola, 1837; Pentatoma albocostatum Spinola, 1852; Pentatoma dimidiaticollis (Spinola, 1852); Pentatoma unidentatum Spinola, 1852; Schaefferella litigiosa Spinola, 1850, Sympiezorhincus tristis Spinola, 1837; and Thoreyella brasiliensis Spinola, 1850. A lectotype is also designated for Chlorocoris tau, putting aside the previous Neotype designation. The preservation status of each specimen is provided.
\end{abstract}

KEY WORDS. Lectotype; taxonomy.

The hemipterological collection of Massimiliano Spinola is preserved in the Museo Regionale de Scienze Naturali (MRSN, Turin, Italy). It includes syntypes of the species originally described by SpInola $(1837,1850,1852)$ along with other specimens and has been fully catalogued by CASALE (1981). Despite its great importance and although beautifully organized and extremely useful, no information is available in the catalog about the current identification and synonyms of the species, since these are listed and named according to the original labels of Spinola accompanying the specimens. In order to fill this gap and contribute with a modern taxonomic treatment, as well as to properly designate lectotypes, the collection has been visited and 140 specimens of Neotropical pentatomids (Asopinae, Discocephalinae, Edessinae and Pentatominae) have been examined and identified. The examination of the specimens at MRSN, in a first trip, was supported by CNPq with grants to J. Grazia and M. Becker during the midsummer of 1996; a second trip made by J. Grazia, in late autumn 1997, was supported by the MRSN.

\section{MATERIAL AND METHODS}

This work presents the species in alphabetical order using the names as written on the original labels. This systematization is adopted for two main reasons: first, this helps to maintain congruence with the catalog of CASALE (1981) and eases future researchers to locate the specimens in the collection. Second, a confuse listing is avoided because many of the species names on the labels used by Spinola were never published, and so are not valid names. When this is the case, the observation "unpublished name" or the actual name published by Spinola follows the species name.
For each species, the following data are provided: name as found on the label, with citation if published by Spinola; the current valid name or an identification provided by the present authors (indicated by "="); when pertinent, after the valid name or identification the citation for synonymy is given; number, gender and condition of the specimens; data found on labels; number of the collection box where the specimen is stored. When we were not able to provide the species identification, we indicated it as most detailed as possible.

The rectangular green labels, which contain the original data on the specimens, are attached to the bottom of the boxes; the green color represents the origin of the material (Neotropical region); in some specimens, a round green label, or a square white label, is attached to the insect pin (Figs 1 and 2).

Digital photographs of eight type specimens are also provided.

\section{TAXONOMY}

Antiteuchus signoreti. Unpublished name

= Antiteuchus tesselatus (Westwood, 1837).

Material examined. One female in fairly good condition. Green label: "Antiteuchus Signoreti [sic], m n. sp., D. Buquet, Brasilia”. Remarks. Besides the general color pattern, the antennal segment I is longer than the II, a distinct character mentioned in Fernandes \& Grazia (2006) to distinguish this species.

Box 7.

Apodiphus torquatus. Unpublished name (Figs 3, 17)

Described as Chlorocoris tau Spinola, 1837: 289.

Material examined. One female in poor condition, with 


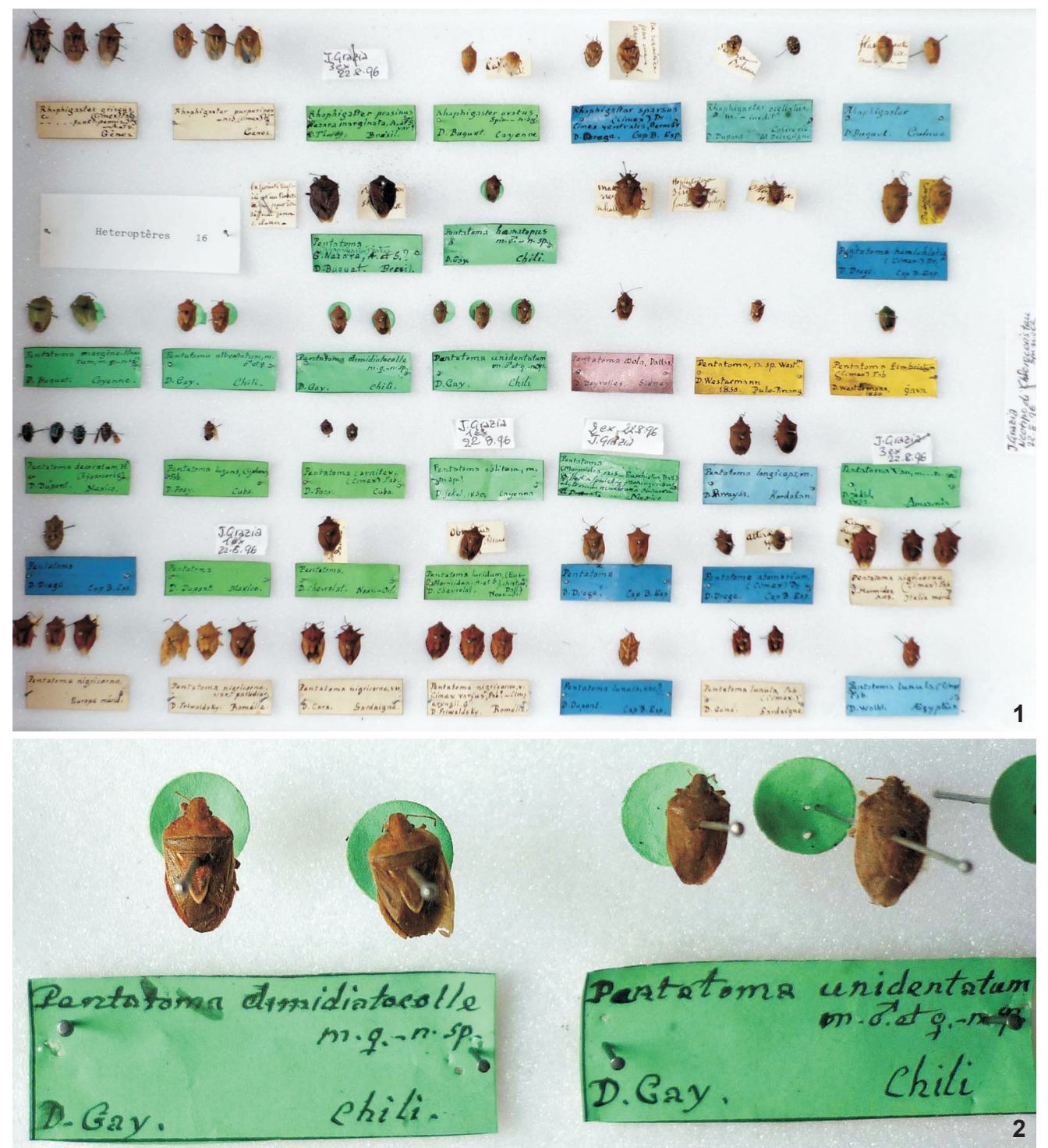

Figures 1-2. Spinola's collection box, Museo Regionale de Scienze Naturali, Turin. (1) Full box; (2) detail of the same box showing lectotype male and female of Pentatoma dimidiatocolle [sic], lectotype male and female of $P$. unidentatum, and the respective green labels.

only the first left antennal segment present, both hemelytra and the apex of the scutellum missing. One female in fairly good condition, with a white label on pin"Chlorocoris complanatus, Dall., (Pentatoma) Guérin, Chlor. tau [sic], m. olim [illegible]”.

Both females with the green labels: "Apodiphus torquatus, (Gastraulax), H. Sch., G. Thelima, A. et S.”.

Remarks. The female in better condition is here designated LECTOTYPE, putting aside the previous neotype designation made by Thомаs (1985: 678), in accordance with the Article 75 of the International Code of Zoological Nomencla- ture (ICZN 1999: 86).

Box 8.

\section{Arma}

= Euschistus (Lycipta) circumfusus Berg, 1883

Material examined. One female in good condition with the green label: "Arma..., D. Buquet, Brésil". One male lacking the pygophore and with the green label with same data as the female.

Box 20. 
Arma longifrons. Unpublished name

= Euschistus (Euschistus) atrox (Westwood, 1837).

Material examined. One female in fairly good condition, labeled "Arma n. sp.? longifrons mihi, D. Buquet; Brésil". Box 20.

\section{Arma nigrolimbata. Unpublished name}

Described as Oplomus nigro-limbatus Spinola, 1852: 122-124.

= Brontocoris nigrolimbatus (Spinola, 1852); Тномаs (1992: 2830).

Material examined. Two females in fairly good condition, each bearing a small round green label "1943". Green label: "Arma nigrolimbata m., 우 $\sigma^{\star}, \mathrm{D}$. Gay; Chili".

Remarks. The specimens probably belong to the typical series of Oplomus nigro-limbatus Spinola, not included in the sample deposited at Muséum National d'Histoire Naturelle (Paris) (Thomas 1992: 30).

Box 20.

Arvelius edessoides Spinola, 1837: 347

= Taurocerus edessoides (Spinola, 1837); Amyot \& Serville (1843: 151).

Material examined. Two females in fairly good condition, lacking part of the antennae. Green label: "Arvelius edessoides, Spin., G. taurocerus, A. et S., D. Buquet; Brésil”. Remarks. Lectotype and paralectotype were designated by GRAZIA $\&$ Barcellos (2005).

Box 13 (13-14).

Arvelius gladiator Spinola, 1837: 346 (Figs 4, 11, 12)

= Arvelius albopunctatus (De Geer, 1773); synonymized by DisTaNT (1880: 82).

Material examined. One female and one male, both in poor condition. Green label: "Arvelius gladiator, Spin., Cimex albopunctatus, Deg., D. Buquet, Brésil".

Remarks. A LECTOTYPE is herein designated for A. gladiator in order to assign a single specimen of this species as the name-bearer and thus, stabilize the nomenclature. There are two specimens labeled as A.gladiator Spinola in the MRSN, none of which is designated as a type. Therefore, the male was selected and a lectotype label was attached to its pin.

Box 13 (13-14).

Arvelius laciniatus Spinola, 1837: 347

= Evoplitus humeralis (Westwood, 1837); synonymized by Амүот $\&$ Serville (1843: 153).

Material examined. Two females in fairly good condition, one lacking the right hemelytron. No labels.

Remarks. The female in better condition is here designated LECTOTYPE for Arvelius laciniatus Spinola, 1837 in order to assign a single specimen of this species as the name-bearer. Box 13 (13-14).
Audinetella bipunctata Spinola, 1850: 128-129 (Figs 6, 18)

= Lincus bipunctatus (Spinola, 1850) in part; ANONYmous (1993: 246-247).

Material examined. One female in good condition labeled "Lincus croupius Rolston, det. L.H. Rolston 1990". Green label: "Audinetella bipunctata m., D. Buquet, Cayenna".

Remarks. This female is here designated LECTOTYPE, considering that there are two females under the same green label (see below) and to assign a single specimen of this species as the name-bearer. RoLsTon (1992: 19-21) proposed for the International Commission of Zoological Nomenclature (ICZN) the suppression of the names Audinetella and A. bipunctata in favor of Lincus and L. croupius. The ICZN decided in favor of Lincus but not of $L$. croupius, which has the status of a junior subjective synonym of L. bipunctatus (ANonymous 1993: 246-247).

Box 16 (16-17).

Audinetella bipunctata Spinola, 1850: 128-129

= Paralincus Rolston, 1983 in part.

Material examined. One female with damaged abdomen labeled "Paralincus sp., det. L.H. Rolston 1990". Green label: "Audinetella bipunctata m., D. Buquet, Cayenna". Box 16 (16-17).

Brachystethus quinquedentatus Spinola, 1837: 344

= Lopadusa (Bothrocoris) quinquedentata (Spinola, 1837); BECKER \& Grazia (1970: 225).

Material examined. One female in fairly good condition, lacking the last two antennal segments of both antennae. Green label: "Brachystethus quinquedentatus, Spin., D. Buquet, Brésil". Box 7.

Cataulax macraspis Spinola, 1837: 339 (Figs 6, 13, 14) = Cataulax eximius (Stål, 1860); synonymized by STÅL (1872: 7).

Material examined. One male in fairly good condition. Antennae missing, except the first segment of right antennae, right hemelytron missing. Green label: "Cataulax macraspis, Spin., D. Buquet, Brésil".

Box 7.

Coryplatus [lapsus calami] laciniatus. Unpublished name = see Remarks.

Material examined. One female. Antennae missing, except the first segment of left antenna. Green label: "Coryplatus laciniatus m. n. sp., Brasilia".

Remarks. The specimen belongs to an unidentified genus of Discocephalini near to Alcippus Stål, 1867 and to Paralcippus Becker \& Grazia, 1986.

Box 7.

Cylindroscelis divaricata. Unpublished name

= Oebalus poecilus (Dallas, 1851).

Material examined. One female. White label: "Mormidea 

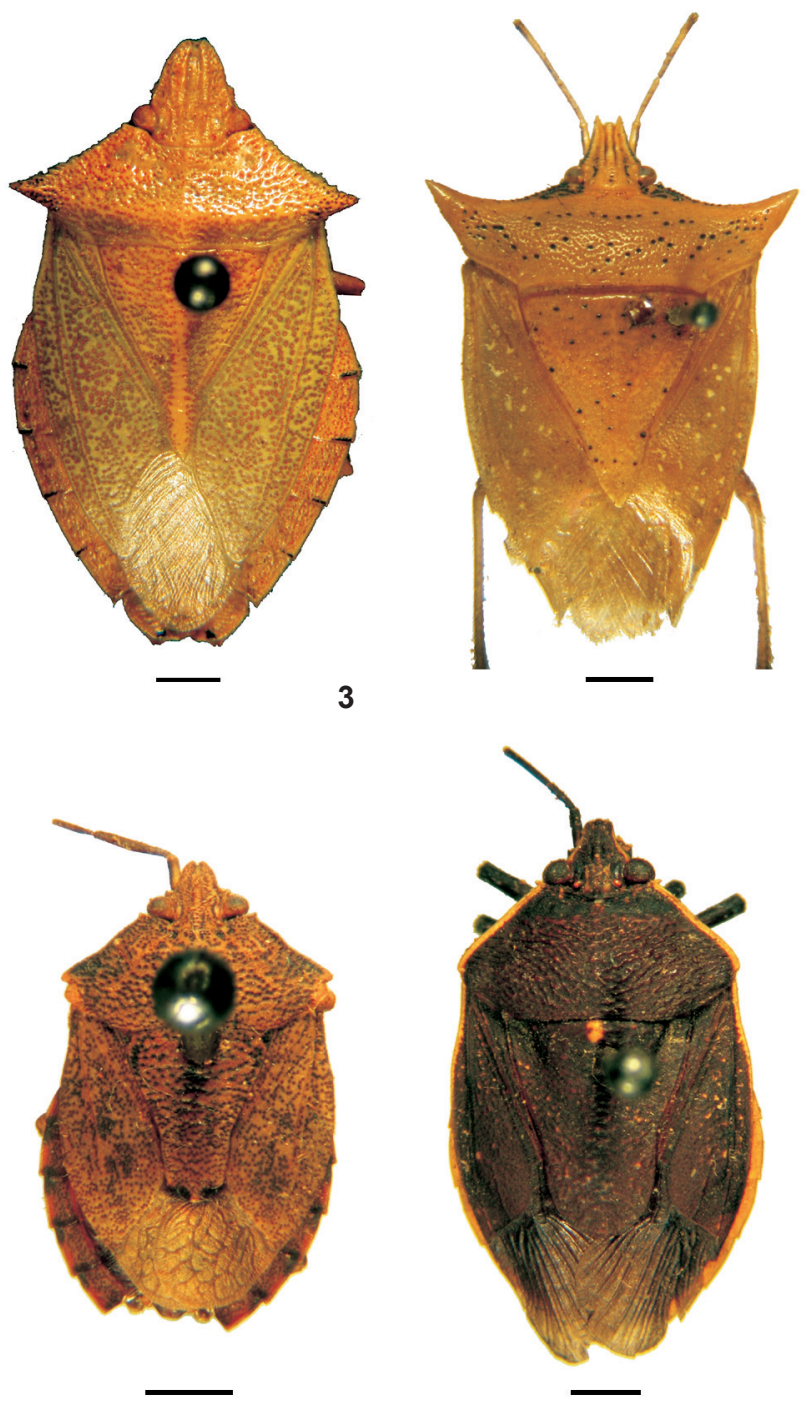

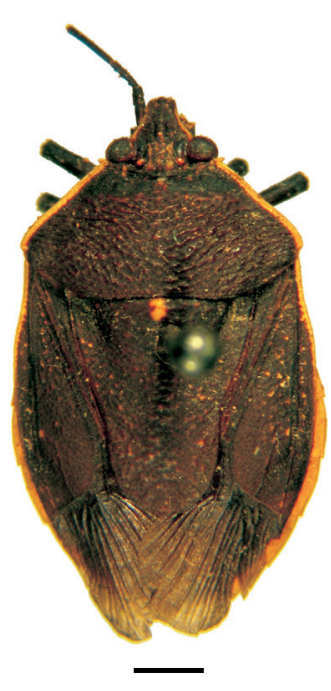

8
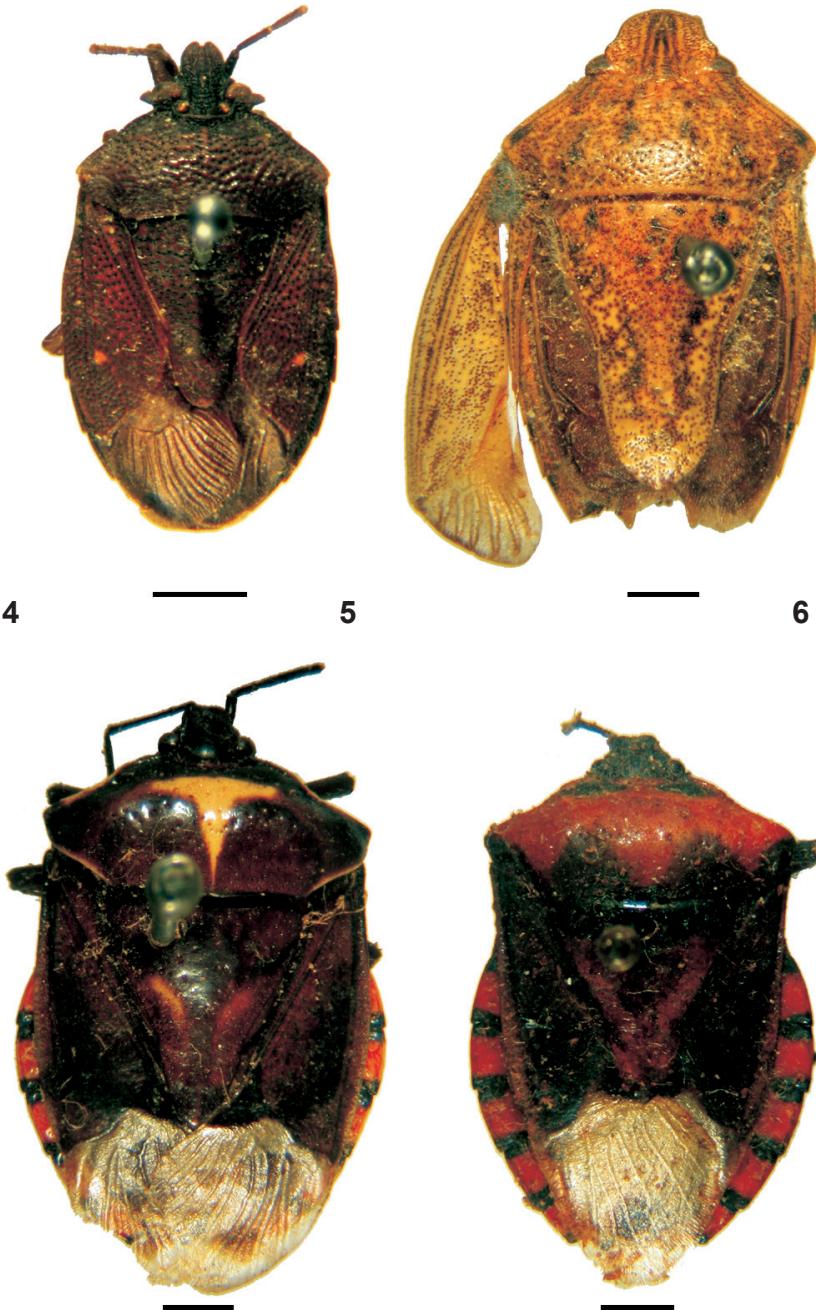

9

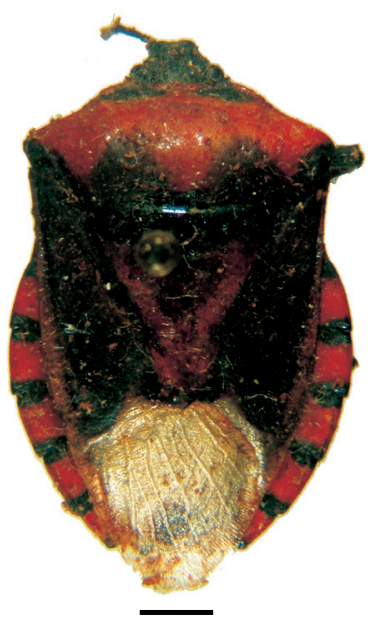

10

Figures 3-10. Types of Neotropical Pentatomidae of the Spinola Collection, Museo Regionale de Scienze Naturali, Turin. Dorsal facies: (3) Chlorocoris tau, female lectotype; (4) Arvelius gladiator, male lectotype; (5) Audinetella bipunctata, female lectotype; (6) Cataulax macraspis, male holotype; (7) Schaefferella litigiosa, male lectotype; (8) Ochlerus cinctus, female lectotype; (9) Vulsirea ancora, female holotype; (10) Vulsirea nigrorubra, female holotype. Scales $=2 \mathrm{~mm}$.

Poecila [sic] Dallas". Green label: "Cylindroscelis divaricata, m. n. sp., D. Buquet, Brasilia".

Box 14 (14-15).

Cylindroscelis eburneata. Unpublished name = Oebalus ypsilongriseus (De Geer, 1773).

Material examined. One female. White label: "Mormidea P. griseus, D. Geer"; small green label: "ㅇ". Green label:
"Cylindroscelis eburneata, m. n. sp, ơ + [sic], Brasilia". Box 14 (14-15).

Cylindroscelis typhoeus. Unpublished name = Oebalus pugnax (Fabricius, 1775); STÅL (1872: 22).

Material examined. Two females. Green label: "Cylindroscelis Typhoeus [sic] (Cimex), Fabr. (Mormidea Dall.), D. Carmine, Carolina". 
Box 14 (14-15) (miscellanea).

Diacus rufescens. Unpublished name

= Dichelops (Neodichelops) furcatus (Fabricius, 1775).

Material examined. Two females in fairly good condition, one labeled "Zalega A. et Serv."; the other labeled "ㅇ” " and with a small green label: "Zalega fursifrons ... [illegible]". Green label: "Diacus rufescens, m. n. sp., D. Buquet, Brasilia".

Box 19.

Dinidor annulipes. Unpublished name

= Dinocoris (Dinocoris) maculatus Laporte, 1832.

Material examined. Two males, one in fairly good condition, left antenna lacking the two last segments; the other in very poor condition. Green label: "Dinidor annulipes ? - o m., Din. maculatus var. ?, Cayenna".

Box 7.

Discocephala lata. Unpublished name

= Phoecia Stål, 1862.

Material examined. One male, lacking three last segments of left antenna, and one female in fairly good condition. Green

label: "Discocephala lata, m., n. sp. ㅇ, , o', D. Buquet - Brasilia". Box 7.

Dynotomarus spectabilis. Unpublished name

= unidentified genus.

Material examined. One male, labium missing, pygophore damaged. Green label: "Dynotomarus spectabilis m., $\sigma^{x}-$ n. c. ineditum 1850, D. Doyrella, Cayenna".

Box 11 (10-11) (miscellanea).

Edessa amazonia. Unpublished name

= see Remarks.

Material examined. Two males in poor condition. Green label: "Edessa amazonia, m., D. Jekel 1851, Amazonia".

Remarks. The specimens probably belong to Edessa meditabunda (Fabricius, 1794).

Box 13 (13-14).

Edessa brevidens. Unpublished name

= Edessa Fabricius, 1803.

Material examined. Two males and one female in fairly good condition. Female with a white label: "Pigoda [sic], A. S."; one of the males with a white label: "sentata Dall". Green label: "Edessa brevidens, Spin. - n. sp. ? $\sigma^{\top}$ 우, D. Buquet, Brésil". Remarks. The males are not conspecific.

Box 12 (12-13).

\section{Edessa incumbens. Unpublished name}

= Taurocerus edessoides (Spinola, 1837).

Material examined. One female in fairly good condition. White label: "Taurocerus edessoides Spin., voir plus [illegible]
Hand [illegible]". Green label: "Edessa incumbens, m. - n. sp.?, D. Jekel 1851, Amazonia".

Box 12 (12-13).

Edessa nigra. Unpublished name

= Edessa Fabricius, 1803.

Material examined. One male and one female in fairly good condition. Female with a white label: "aceratodes [sic] fulvipes Dall."

Box 13 (13-14)

Edessa rugicollis. Unpublished name

= Edessa polita (Lepeletier \& Serville, 1825).

Material examined. One female in fairly good condition. Only the first three antennal segments are present in the right antenna. Small white label below the insect. Green label: "Edessa rugicollis, Spin. - n. sp.?, D. Buquet, Brésil".

Box 12 (12-13)

Edessa strigulosa. Unpublished name

= Edessa Fabricius, 1803.

Material examined. One female in fairly good condition. Green label: "Edessa strigulosa, m. n. sp., Cayenna". Box 13 (13-14).

\section{Epipedus histrio Spinola, 1837: 315-316}

Material examined. Two females, one in poor condition and the other in fairly good condition. White label: "Epipedus histrio Spin., D. Buquet Brésil (cotype)". Red label: "Lectotype Epipedus histrio Spinola, L. H. Rolston 1986". Green label: "Epipedus histrio, Spin., D. Buquet Brésil".

Box 19 (19).

Loxa flavicollis (Drury, 1773)

Material examined. Two females, one in very good condition and one in fairly good condition. Two females in very poor condition. White label: "Loxa flavicollis, var. virescens, Brasilia". Green label: "Loxa flavicollisi, (Cimex) Drury et Dall. Chlorocoris virescens m. oliva, Brasilia".

Box 19 (19).

Macropygium atrum Spinola, 1837: 288.

= Macropygium reticulare (Fabricius, 1803); Dallas (1851: 150, 158-159).

Material examined. One female in fairly good condition; three males, two in good condition and one in poor condition, lacking the pygophore. Green label: "Macropygium atrum Spin. $\sigma^{\top}$ ㅇ, D. Buquet Brésil".

Remarks. Spinola (1837: 288) described M. atrum based on one male collected by M. Dupont in Brazil and stated that the female of the species was unknown.

Box 18 (18)

Ochlerus cinctus Spinola, 1837: 295 (Figs 8, 19)

Material examined. Two females in fairly good condi- 

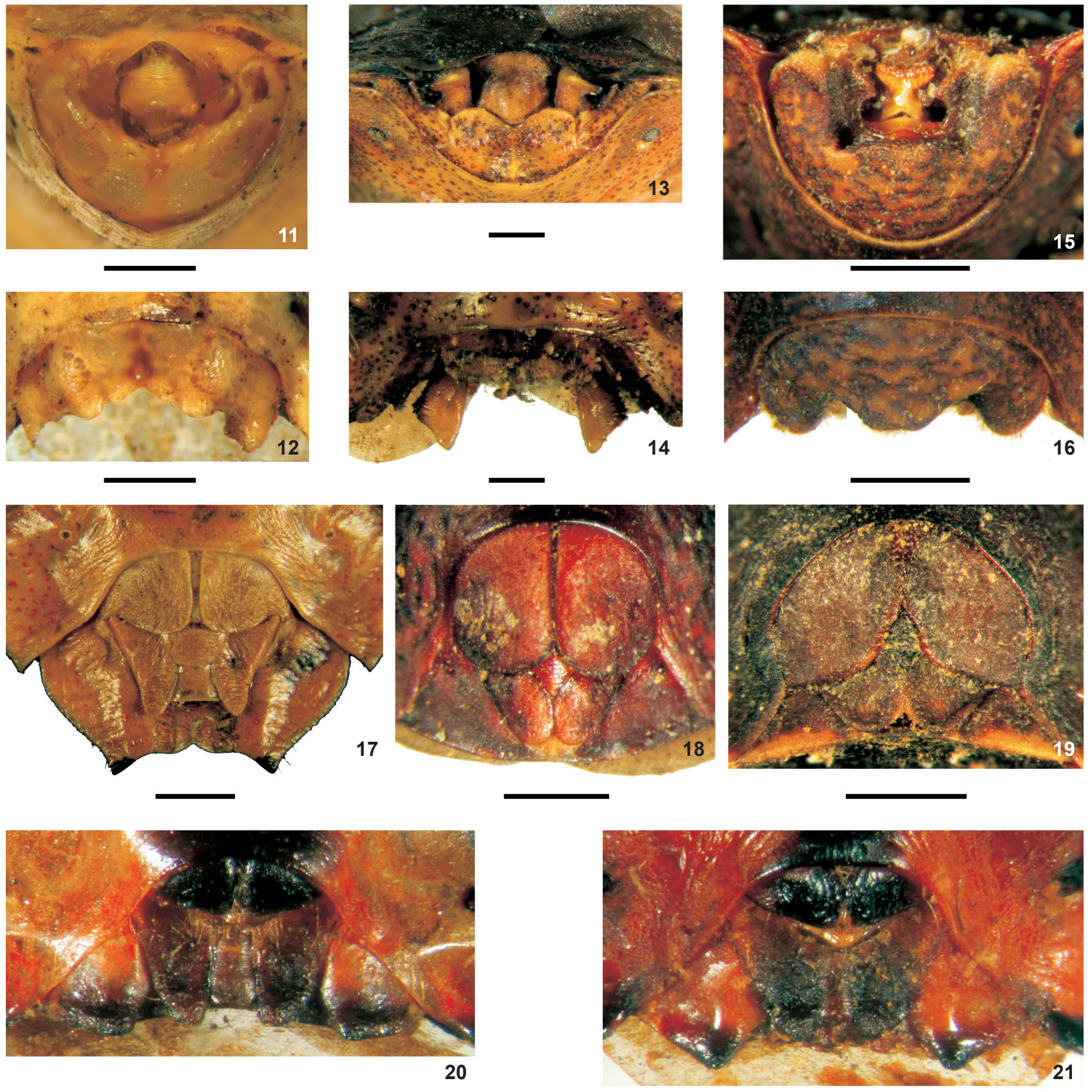

20 21

Figures 11-21. Types of Neotropical Pentatomidae of the Spinola Collection, Museo Regionale de Scienze Naturali, Turin. Genitalia: (11-12) Arvelius gladiator, male lectotype: (11) posterior view; (12) ventral view; (13-14) Cataulax macraspis, male holotype: (13) posterior view; (14) ventral view; (15-16) Schaefferella litigiosa, male lectotype: (15) posterior view; (16) ventral view; (17) Chlorocoris tau, female lectotype, ventral view; (18) Audinetella bipunctata, female lectotype, posteroventral view; (19) Ochlerus cinctus, female lectotype, posteroventral view; (20) Vulsirea ancora, female holotype, ventral view; (21) Vulsirea nigrorubra, female holotype, ventral view. Scales $=1 \mathrm{~mm}$. 
tion. Green label: "Ochlerus cinctus var., D. Buquet Brasilia". Remarks. One female is here designated LECTOTYPE, considering there are two females under the same green label and to assign a single specimen of this species as the namebearer. SPINOLA (1837: 295) mentioned a female and a male in his description; however, the male has not been located. Box 18 (18).

Ochlerus cinctus Spinola, 1837: 295

Material examined. Three females in good condition.

Green label: "Ochlerus cinctus Spin. + , Menipha brunea, A. et S.?, D. Buquet Brésil".

Remarks. These specimens are not cospecific, two females belong to $O$. cinctus and one female belongs to an unknown species of Paralincus.

Box 18 (18).

\section{Pentatoma}

= Euschistus (Euschistus) biformis Stål, 1862.

Material examined. One male. Green label: "Pentatoma,

D. Dupont Mexico".

Box 15 (15-16).

Pentatoma

= Euschistus (Mitripus) acutus Dallas, 1851 in part.

Material examined. One female. White label: "Pentatoma... (Mormidea, A. S. - Euschistus, Dall.), Differt a especies prope..., D. Dupont, Mexico". Box 15 (15-16).

Pentatoma

= Euschistus (Euschistus) crenator (Fabricius, 1794) in part.

Material examined. One female. White label: "Pentatoma... (Mormidea, A. S. - Euschistus, Dall.), Differt a especies prope..., D. Dupont, Mexico". Box 15 (15-16).

Pentatoma

= Pellaea stictica (Dallas, 1851).

Material examined. Three females, two in fairly good condition and one in poor condition. The first two with a white label: "La pointe ventral ici est un tubercle qui peut ètre difficile pour le classer". The third with a white label: "Raphigaster Dall.". Green label: "Pentatoma... (Raphigaster) Dallas, G. Nezara A. et S.?, D. Buquet, Brésil".

Box 15 (15-16).

\section{Pentatoma}

= Euschistus (Euschistus) atrox (Westwood, 1837).

Material examined. Three females in fairly good condition, one labeled " $\sigma$ ", one without white label and one labeled "o " and with a white label: "Euschistus atrox Dall.?". Green label: "Pentatoma (A), D. Buquet, Cayenne". Box 16 (16-17).

\section{Pentatoma}

= Euschistus (Euschistus) crenator (Fabricius, 1794).

Material examined. One male in fairly good condition with a white label: "G. Euschistus, Dall., Differe de A par les epines du protorax et le nervures des la membrane des ailes inferieures". One female in poor condition with a white label: "Pustulata [sic] Pall. Beauv.". Green label: "Pentatoma (A) var.?, D. Buquet, Cayenne". Box 16 (16-17).

\section{Pentatoma}

= Euschistus (Lycipta) triangulator (Herrich-Schaeffer, 1842).

Material examined. One male labeled " $\sigma^{x}$ " and with a white label: "Euschistus apicalis, Dall - Remarque l'innervation de la membrane elitrale". Green label: "Pentatoma, D. Buquet, Cayenne".

Box 16 (16-17).

\section{Pentatoma}

= Thyanta $($ Thyanta $)$ perditor (Fabricius, 1794).

Material examined. One female in poor condition. White label: "Pentatoma, Coll. Bosc., Antilla [sic]". Green label: "Dimidiatum, v.?, membrane imaculata". Box 17 (17).

\section{Pentatoma}

= Roferta marginalis (Herrich-Schaeffer, 1836).

Material examined. One male and one female in fairly good condition, the latter with a white label: "Pentatoma nitida, Hope marginella H. S.". Green label: "Pentatoma... Brasilia".

Box 17 (17).

\section{Pentatoma albeastatum. Unpublished name}

Described as Pentatoma albo-costatum Spinola, 1852: 132.

Material examined. One male and one female in fairly good condition, labeled " $\sigma$ " and "ㅇ " respectively. Male with a small, round green label, "1943". Green label: "Pentatoma albestatum [sic], m. ơ et 9 ., D. Gay, Chili".

Remarks. The male is here designated LECTOTYPE, considering there are two specimens under the same green label and to assign a single specimen of this species as the name-bearer. Box 15 (15-16).

Pentatoma apicicorne Spinola, 1852: 131

= Chinavia apicicornis (Spinola, 1852); Grazia \& Schwertner (2008: 233).

Material examined. Two females in very poor condition, one with a small round green label "943". White label: "Pentatoma apicicorne m. n. sp., o $\sigma^{\top}$ ㅇ, D. Gay, Chili". Box 17 (17).

Pentatoma aurantiaca. Unpublished name Described as Arocera aurantiaca Spinola, 1837: 318. 
Figure 1 ( $3^{\text {rd }}$ row, $3^{\text {rd }}$ and $4^{\text {th }}$ specimens, from left to right). = Arocera acroleuca (Perty, 1833) in part; SignoRet (1851: 338).

Material examined. One female in very poor condition and one male in fairly good condition. Green label: "Pentatoma aurantiaca, (Arocera) m., D. Buquet, Brésil".

Box 18 (18).

\section{Pentatoma aurantiaca. Unpublished name}

Described as Arocera aurantiaca Spinola, 1837: 318. = Arocera aequinoxialis Westwood, 1837 in part; revalidated by RIDER (1992: 105).

Material examined. One male in fairly good condition, with a white label "arocera [sic] Spin., oedosoma a et s [sic], acroleucum Perty, aurantiacum Spin., pourquoi n'a plus conserver arocera qui eu De Vour [illegible]". Green label: "Pentatoma aurantiaca, (Arocera) m., D. Buquet, Brésil".

Box 18 (18).

\section{Pentatoma consobrino. Unpublished name}

= Sibaria armata (Dallas, 1851).

Material examined. Two males and two females in good condition. One male and one female labeled " $\sigma$ " and "우", respectively. One female with a white label: "Mormidea armata Dall.". Green label: "Pentatoma consobrinum (Mormidea) A. S., Pentamota [sic] latum H. Sch. \& Hahn, angulis pronoti [three illegible words], D. Buquet, Cayenne".

Box 16 (16-17).

Pentatoma dimidiatocolle [sic] Spinola, 1852: 133-134

= Acledra dimidiaticollis (Spinola, 1852); STÅL (1867: 528). and 2 .

Figures 1 ( $3^{\text {rd }}$ row, $5^{\text {th }}$ and $6^{\text {th }}$ specimens, from left to right)

Material examined. One male and one female in fairly good condition, with small green label "1943". Male labeled " $\mathrm{o}^{\mathrm{x}}$. Green label: "Pentatoma dimidiaticolle [sic] m. o n. sp., D. Gay, Chili".

Remarks. Faundéz \& Verdejo (2009) mentioned two females of $P$. dimidiaticolle [sic] deposited at MRSN, apparently due to a misinformation provided by the Museum. The male is here designated LECTOTYPE, considering there are two specimens under the same green label and to assign a single specimen of this species as the name-bearer.

Box 15 (15-16).

\section{Pentatoma dissimile}

= Palomena prasina (Linnaeus, 1761).

Green label: "Pentatoma dissimile, haud diversum, D. Deyrolles, Brasilia".

Remarks. This specimen is conspecific with other four of the same box collected in Italy. Although the original label indicates the Neotropical Region, $P$. prasina is in fact a Palearctic species.

Box 16 (16-17).

\section{Pentatoma eburneiger. Unpublished name}

= Podisus aenescens (Stål, 1860).

Material examined. One male in fairly good condition and one female in very poor condition with a white label: "Nec libre virs à mettre dans les asopides". Green label: "Pentatoma eburneiger, m., D. Jekel 1851, Amazonia".

Box 18 (18).

Pentatoma formosa. Unpublished name

= Vulsirea formosa Dallas, 1851.

= Pharypia pulchella (Drury, 1782); STÅL (1872: 40).

Material examined. Two females, one in poor condition and one in fairly good condition with a white label: "Vulsirea concentricus Drury". Green label: "Pentatoma formosa Dall.

(Vulsirea) 우, G. Nezara, A. et S., D. Ghiliani, Para 1866".

Box 18 (18).

Pentatoma formosum. Unpublished name

= Vulsirea formosa Dallas, 1851 .

= Pharypia pulchella (Drury, 1782); STÅL (1872: 40).

Material examined. One male and one female in fairly good condition. Green label: "Pentatoma formosum var. $\sigma^{x}+, \mathrm{D}$. Buquet, Brasilia".

Box 18 (18).

Pentatoma guttatipenne. Unpublished name

= Thyanta Stål, 1862.

Material examined. One female in fairly good condition. Green label: "Pentatoma guttatipenne, m. n. sp.?, D. Dupont, Mexico".

Box 17 (17).

Pentatoma haematopus Spinola, 1852: 136

= Acledra hematopa (Spinola, 1852); STÅL (1872: 32).

Fig. 1 ( $2^{\text {nd }}$ row, $3^{\text {rd }}$ specimen from left to right).

Material examined. One male in poor condition with a small, round green label, "1943". Green label: "Pentatoma haematopus m. $0^{x}$ - n. sp., D. Gay, Chili".

Box 15 (15-16).

Pentatoma longiceps. Unpublished name

= Tibraca limbativentris Stål, 1860.

Material examined. Two males in good condition. Green

label: "Pentatoma longiceps - m., D. Parreyss, Kordofan".

Remarks. Although the original label indicates the Ethiopic

Region, T. limbativentris is in fact a Neotropical species. Box 15 (15-16).

\section{Pentatoma longigena. Unpublished name}

= Euschistus Dallas, 1851.

Material examined. One female in poor condition. White label: "Pentatoma longigena, m. n. sp., (Mormidea, A. S - Euschistus, Dallas) - La peut antica emargination, D. Dupont, Mexico". Box 16 (16-17). 


\section{Pentatoma oblitum. Unpublished name}

$=$ see Remarks.

Material examined. One female in fairly good condition. Green label: "Pentatoma oblitum, m. n. sp.?, D. Jekel, 1850, Cayenna".

Remarks. The specimen belongs to an unidentified species of the group of Hypatropis Bergroth, 1891.

Box 15 (15-16).

Pentatoma quadrigutticolis. Unpublished name

= see Remarks.

Material examined. One female in good condition. Green label: "Pentatoma quadrigutticolis, m. - n. sp. - G. Mormidea A. S. et Dall., D. Ghiliani, 1866, Para".

Remarks. The specimen belongs to Mormidea Amyot \& Serville, 1843, probably Mormidea (Mormidea) maculata Dallas, 1851.

Box 16 (16-17).

Pentatoma spectabilis. Unpublished name

= Pharypia Stål, 1861.

Material examined. One male in poor condition. White label: "Pentatoma spectabilis, (Cimex) Klug ox, D. Klug, Mexico". One female in good condition. Green label: "Pentatoma spectabilis 우, G. Nezara, A et S., D. Klug, Mexico".

Box 18 (18).

Pentatoma unidentatum Spinola, 1852: 134

= Oenopiella unidentata (Spinola, 1852); STÅL, 1867: 529 (as Oenopia unidentata).

Figures 1 ( $3^{\text {rd }}$ row, $7^{\text {th }}$ to $9^{\text {th }}$ specimens, from left to right) and 2 .

Material examined. One male and two females, each one with a small green label "1943". Green label: "Pentatoma unidentatum m. $\sigma^{\top}$ et 9 - n. sp., D. Gay, Chili”.

Remarks. The male is here designated LECTOTYPE, considering there are three specimens under the same green label and to assign a single specimen of this species as the namebearer.

Box 15 (15-16).

\section{Pentatoma vau. Unpublished name}

= Mormidea Amyot \& Serville, 1843.

Material examined. Three females in fairly good condition. Green label: "Pentatoma vau m. n. sp., D. Jekel 1852, Amazonia".

Box 15 (15-16).

\section{Pentatoma ypsilon (Linnaeus, 1758)}

= Mormidea (Mormidea) v-luteum (Lichtenstein, 1796) in part.

Material examined. One female in good condition with a white label "crosseipes H. Sch.". Green label: "Pentatoma ypsilon, var., D. Buquet, Bresil".
Pentatoma ypsilon (Linnaeus, 1758)

= Mormidea (Mormidea) ypsilon (Linnaeus, 1758); Dallas (1851: 211) in part.

Material examined. One male in good condition. Green label: "Pentatoma ypsilon, var., D. Buquet, Bresil".

Box 16 (16-17).

Phyllocheirus

= Heteroscelis servillei Laporte, 1833.

Material examined. One female in fairly good condition. White label: "Phyllocheirus, D. Signoret, Pernambuco".

Box 20 (20).

Platencha murina. Unpublished name

= Tibilis subconspersa Stål, 1860.

Material examined. One male. Green label: "Platencha murina, m. (inedita), D. Buquet, Brésil".

Box 13 (13-14).

Proxys hastator (Fabricius, 1798)

= Berecynthus hastator (Fabricius, 1794); RIDER \& ROLSTON (1995: 847).

Material examined. One female in poor condition. Green label: "Proxys hastator (Cymex Fab.), Pr. crenatus A. S., D. Deyrolles, Cayenna".

Box 14 (14-15) (miscellanea).

Proxys victor (Fabricius, 1775)

= Proxys albopunctulatus (Palisot de Beauvois, 1805).

Material examined. Two males in good condition. Green label: "Proxys victor, Spin., Cimex victor Fab., Brésil".

Remarks. These are not the types, since Spinola described Proxys for Cimex victor Fabricius.

Box 14 (14-15).

Raphigaster ovatus. Unpublished name

= Piezodorus guildinii (Westwood, 1837).

Material examined. One male in good condition; one specimen lacking the abdomen and with a white label: "Catinus Dall.". Green label: "Raphigaster ovatus Spin. - n. sp.?, D. Buquet, Cayenna".

Box 15 (15-16)

Raphigaster prasinus. Unpublished name

= Chinavia esmeralda (Rolston, 1983) in part.

Material examined. One male with damaged pygophore. Green label: "Raphigaster prasinus Spi., Nezara marginata, A. et S. var.?, D. Torey, Brésil". Box 15 (15-16).

Raphigaster prasinus. Unpublished name

= Chinavia herbida (Stål, 1859) in part.

Material examined. One female and one male, the latter with a white label: "Chlorocephala Hope". Green label: 
"Raphigaster prasinus Spi., Nezara marginata, A. et S. var.?, D. Torey, Brésil".

Box 15 (15-16).

Schaefferella retipennis. Unpublished name (Figs 7, 15, 16)

Described as Schaefferella litigiosa Spinola, 1850: 128.

= Schaefferella incisa (Herrich-Schäffer, 1839); STÅL (1872: 12).

Material examined. One male and one female in fairly good condition; another specimen lacking the abdomen. Green label: "Schaefferella retipennis, m. $\sigma^{\top}$ ㅇ , D. Buquet, Brasilia".

Remarks. The male is here designated LECTOTYPE, considering there are three specimens under the same green label and to assign a single specimen of this species as the name-bearer. Box 18 (18).

\section{Sympiezorhincus tristis Spinola, 1837: 286-287}

Material examined. Two males, one with the abdomen opened and the pygophore glued on a separate label, the other in fairly good condition, antennal segments missing except for the first segment of the left antenna. Green label: "Sympiezorhincus tristis Spin., Rachava orbicularis Amyot \& Serville, D. Buquet, Brésil".

Remarks. SPINOLa (1837: 287) stated that the male of the species was unknown. He certainly misinterpreted the sex of the specimens. The male keeping the pygophore in the abdomen is here designated LECTOTYPE, considering there are two specimens under the same green label and to assign a single specimen of this species as the name-bearer.

Box 7.

Thoreyella brasiliensis Spinola, 1850: 120-121

Material examined. One female in good condition; one male with the appendages in poor condition. Green label: "Thoreyella brasiliensis, m. - inedita, D. Thorey, Brésil".

Remarks. The male is here designated LECTOTYPE, considering there are two specimens under the same green label and to assign a single specimen of this species as the namebearer.

Box 13 (13-14).

Thynacantha chilensis. Unpublished name

Described as Oplomus chilensis Spinola, 1852: 124

= Tylospylus chilensis (Spinola, 1852); STÅL (1870: 53).

Material examined. Two males labeled "1943" and one female labeled "945" and "ㅇ", all in fairly good condition; white label: "Thynacantha chilensis m. (Arma) oli... [illegible], D. Gay, Chile". One male labeled "945"; one female and one specimen lacking the abdomen, both labeled "1943"; white label: "Thynacantha chilensis var, D. Gay, Chili". Two males labeled "1943", one of them labeled " $\sigma$ " and the other with a small white label: "S. Rosa". One female lacking the abdomen labeled "1943" and "o⿱"; white label: "Thynacantha chilensis var., D. Gay, Chili".
Remarks. The two males and the female, mentioned in the beginning of the material examined, probably belong to the typical series of Oplomus chilensis Spinola, not included in the sample deposited at Muséum National d'Histoire Naturelle (Paris) (Тномаs 1992: 116).

Box 20 (20).

Thynacantha nigricornis. Unpublished name.

= Podisus nigrispinus (Dallas, 1851).

Material examined. One female in fairly good condition. Green label: "Thynacantha nigricornis m. n. sp.?, D. Buquet, Brasilia".

Box 20 (20).

\section{Thynacantha rufimargo. Unpublished name}

= Podisus nigrispinus (Dallas, 1851).

Material examined. One female in fairly good condition. Green label: "Thynacantha rufimargo m. n. sp.?, D. Deyrolles, Brasilia".

Box 20 (20).

\section{Thynacantha salebrosa. Unpublished name}

= Podisus nigrispinus (Dallas, 1851).

Material examined. One female in poor condition. Green label: "Thynacantha salebrosa m. n. sp.?, D. Buquet, Brasilia". Box 20 (20).

Thynacantha tabida. Unpublished name

= Podisus nigrispinus (Dallas, 1851).

Material examined. Two males, one lacking the pygophore and labeled " $\sigma$ ", the other labeled "ㅇ". Green label: "Thynacantha tabida m - n. sp., D. Buquet, Brasilia".

Box 20 (20).

Tropicoris chlorizans. Unpublished name

= Acledra fraterna (Stål, 1859).

Material examined. One female in fairly good condition, only the first antennal segment is preserved. White label: "Pentatoma". Green label: "Tropicoris chlorizans, m. - inedit.?, D. Buquet, Brésil".

Box 19 (19).

Vulsirea nigro-rubra [sic] Spinola, 1837 (Figs 9, 20)

Described as Vulsirea ancora Spinola, 1837: 351.

= Vulsirea violacea (Fabricius, 1803); revalidated by Kormilev, 1951: 85-86.

Material examined. One female in good condition. Green label: "Vulsirea nigro-rubra [sic], var. ancora, Spin [illegible], Cuba". Box 14 (14-15).

Vulsirea nigro-rubra [sic] Spinola, 1837: 351 (Figs 10, 21) Material examined. One female. Green label: "Vulsirea nigro-rubra [sic], Spin., Pent. Poey, Guér. ined., D. Poey, Cuba". Box 14 (14-15). 
Vulsirea nigro-rubra [sic] Spinola, 1837: 351.

Material examined. One female in good condition. Green

label: "Vulsirea nigro-rubra [sic], var. D. Dupont, Mexico". Box 14 (14-15).

Zalega furcifrons Amyot \& Serville, 1843: 139.

= Dichelops furcifrons (Amyot \& Serville, 1843); MAYR (1866: 52).

Material examined. One female with a white label: "Zalega

furcifrons A. et S.".

Box 20 (20).

\section{ACKNOWLEDGEMENTS}

We are very grateful to the Director of the Museo Regionale di Scienze Naturali, G. Pagliano, and to P.L. Scaramozzino for the access to the collection and the loaning of material from MRSN; his friendship (and also of his wife, M. Generani) will never be forgotten. To L. Picciau (Università di Torino, Facoltà di Agraria) for sending the digital photographs of one box from Spinola's collection, as well as additional information. We are also thankful to M. Becker for her great contribution to the accomplishment of this project. Finally, the reviewers whose suggestions helped to improve the manuscript. JG thanks the CNPq for a research fellowship.

\section{LITERATURE CITED}

Амyot, J.B. \& A. Serville. 1843. Histoire Naturelle des Insectes. Hémiptères. Paris, Librairie Encyclopédique de Roret, 681p.

Anonymous. 1993. Lincus Stål, 1867 (Insecta, Heteroptera): conserved; L. croupius Rolston, 1983: specific name not conserved. Bulletin of Zoological Nomenclature 50 (3): 246-247.

Becker, M. \& J. Grazia. 1970. Sôbre os gLðneros Lopadusa Stal e Bothrocoris Mayr (Hemiptera, Pentatomidae, Pentatomini). Revista Brasileira de Biologia 30 (2): 217-232.

Casale, A. 1981. Cataloghi. II - Collezione Emitterologica di Massimiliano Spinola. Turin, Museo Regionale di Scienze Naturali, 120p.

DALLAS, W.S. 1851. List of the specimens of hemipterous insects in the collection of the British Museum. Part 1. London, Trustees of the British Museum, 368p.

Distant, W.L. 1880. Insecta. Rhynchota, Hemiptera-Heteroptera, p. 1-88. In: F. D. Godman \& O. SALVIN (Eds). Biologia Centrali - Americana. London, R. H. Porter, vol. 1, XX+462p.

Faundéz, E.I. \& L.M. Verdejo. 2009. The genus Acledra Signoret, 1864 (Hemiptera: Heteroptera: Pentatomidae) in Chile. Zootaxa 2147: 49-58.

Fernandes, J.A.M. \& J. GraZia. 2006. Revisão do gênero Antiteuchus Dallas (Heteroptera, Pentatomidae, Discocephalinae). Revista Brasileira de Entomologia 50 (2): 165-231.

Grazia, J. \& A. Barcellos. 2005. Revision of Taurocerus (Heteroptera, Pentatomidae, Pentatomini). Iheringia, Zoologia 95 (2): 173-181.
Grazia, J. \& C. F. Schwertner. 2008. Pentatomidae e Cyrtocoridae, p. 223-234. In: L.E. Claps, G. Debandi, \& S. Roig-Juñent (Eds). Biodiversidad de Artrópodos Argentinos. Mendoza, Sociedad Entomologica Agentina, vol. 2.

ICZN (International Commission on Zoological Nomenclature) 1999. International Code of Zoological Nomenclature. London, The International Trust for Zoological Nomenclature, $4^{\text {th }}$ ed., 306p.

Kormilev, N.A. 1951. Sobre los géneros Vulsirea Spinola (1837), Ramosiana Kormilev (1950) y Adoxoplatys Breddin (1903), con la descripción de tres especies nuevas (Hemipt. Pentat.). Revista de la Sociedad Entomológica Argentina 15: 8395.

MAYR, G.L. 1866. Hemiptera. In: Reise der österreichischen Fregatte Novara um die Erde in den Jahren 1857, 1858, 1859, unter den Befehlen des Commodore B. von Wüllerstorf-Urbair. II, Abt. 1. Wien, Zoologischer theil, 204p.

Rider, D.A. 1992. Revision of Arocera Spinola, with the description of two new species (Heteroptera: Pentatomidae). Journal of the New York Entomological Society 100 (1): 99-136.

Rider, D.A. \& L.H. Rolston. 1995. Nomenclatural changes in the Pentatomidae (Hemiptera-Heteroptera). Proceedings of the Entomological Society of Washington 97 (4): 845-855.

Rolston, L.H. 1992. Lincus Stål, 1867 (Insecta, Heteroptera): Proposed conservation, and Lincus croupius Rolston, 1983: Proposed conservation of the specific name. Bulletin of Zoological Nomenclature 49 (1): 19-21.

Signoret, V.A. 1851. Description de nouvelles espèces d'Hémiptères. Annales de la Société Entomologique de France 9 (2): 329-348.

SPINOLA, M. 1837. Essai sur les genres d'insectes appartenants à l'ordre des Hémiptères, Lin. ou Rhyngotes, Fab. et à la section des Hétéroptères, Dufour. Genoa, Yves Graviers, 383p.

Spinola, M. 1850. Di alcuni generi d'insetti artroidignati nuovamente proposti dal socio attuale Signor Marchese Massimiliano Spinola nella sua tavola sinottica di questo ordine che precede la presente memoria. Memorie della Societa Italiana delle Scienze residente in Modena 25 (1): 61-138.

Spinola, M. 1852. Hemipteros, p. 113-320. In: C. Gay (Ed.). Historia fisica y politica de Chile. Zoologia. Paris, Maulde y Renou, vol. 7.

STÅL, C. 1867. Bidrag till hemipterernas systematik. Öfversigt af Kongliga Vetenskaps-Akademiens Förhandlingar 24 (7): 491-560.

STÅL, C. 1870. Enumeratio Hemipterorum 1. Kongliga Svenska Vetenskaps-Akademiens Handlingar 9 (1): 1-232.

STÅL, C. 1872. Enumeratio Hemipterorum 2. Kongliga Svenska Vetenskaps-Akademiens Handlingar 10 (4): 1-159.

THomAs, D.B. 1985. Revision of the genus Chlorocoris Spinola 
(Hemiptera: Pentatomidae). Annals of the Entomological Society of America 78 (5): 674-690.

Thomas, D.B. 1992. Taxonomic Synopsis of the Asopine
Pentatomidae (Heteroptera) of the Western Hemisphere. Thomas Say Monograph 16. Lanham, Entomological Society of America, 156p.

Submitted: 25.VIII.2009; Accepted: 29.III.2010.

Editorial responsibility: Gabriel L.F. Mejdalani

ZOOLOGIA 27 (3): 413-424, June, 2010 